\title{
In medium effect on charmonium states using potential model
}

\section{Arpit Parmar}

Department of Physics, Sardar Patel University, Vallabh Vidyanagar-388120, India. E-mail: arpitspudyahoo.co.in

\section{Bhavin Patel}

Department of Physical Sciences, P D Patel Institute of Applied Sciences, Changa, India. azadpatel2003dyahoo.co.in

\section{P C Vinodkumar}

Department of Physics, Sardar Patel University, Vallabh Vidyanagar-388 120, India.

pothodivinodevahoo.com

We present the quarkonia correlators for charmonium systems in the pseudoscalar, vector and scalar channels. For the description of the spectral function we adopt the temperature dependant colour screening potential of the power law form. The spectroscopic parameters defined from the model are employed in the spectral functions to compute the quarkonia correlators. We find considerable medium modifications to the effective masses of the charmonium states. These modifications are then reflected in the computed correlators. The general behaviour of correlators in the vector and scalar channel are in accordance with the latest lattice results. Our analysis provides the dissociation temperature of these charmonium states at $T \sim 1.2 T_{c}$.

XV International Conference on Hadron Spectroscopy

4-8/11/2013

Nara, Japan

\footnotetext{
*Speaker.
} 


\section{Introduction}

The recent study on the spectroscopy of charmonia [W] based on potential models has revealed systematic variations in the confinement strength of the quark-antiquark interaction as the quarkonia gets excited to higher levels. Such state dependant variations of the string tension of the confining part of the potential can be understood in terms of the medium effects. It includes survival probability of the bound states as a function of temperature, in-medium transport properties of the deconfined quarks etc. To incorporate the medium effects on the binding energy of quarkonia, one generally introduces a temperature dependant exponential screening factor in the coulomb part as well as similar terms to the confining part of the potential [四]. The stability of heavy quarkonia states above deconfinement temperature is one of the important topic of interest at the several relativistic heavy ion collider (RHIC) experiments [ [ [ [ []]. Thus, study of production and decay of charmonium states at finite temperature play an important role towards our understanding of the quark-antiquark dynamics in a hot and dense strongly interacting matter.

The dissociation of heavy quarkonia in hot QCD medium can very well understand with the help of correlators and spectral functions. However, earlier studies of correlators (lattice QCD and potential models) show disagreement with each other. We introduce in the next section the spectral functions and define the quarkonia correlators in terms of the spectral function.

\section{Spectral functions and quarkonia correlators}

The temperature dependence of the meson correlators can provide information about the fate of quarkonia states above deconfinement. The imaginary time Euclidean correlation functions of meson currents $G(\tau, T)$ are reliably calculated as [四],

$$
G(\tau, T)=\int d \omega \sigma(\omega, T) K(\tau, \omega, T)
$$

Where, $\sigma(\omega, T)$ is the zero temperature spectral function and the kernel $K(\tau, \omega, T)$ is given by [四],

$$
K(\tau, \omega, t)=\frac{\cosh \left(\omega\left(\tau-\frac{1}{2 T}\right)\right)}{\sinh \left(\frac{\omega}{2 T}\right)}
$$

In lattice QCD the spectral function $\sigma(\omega)$ are generally extracted from the meson correlators using the maximum entropy method [ [ $⿴ 囗 ⿱ 一 一)$. While, the phenomenological models employ the spectral function to compute the quarkonia correlators. Following ref [ [ ] ] the spectral function is written as,

$$
\sigma(\omega)=\sum_{i} 2 M_{i} F_{i}^{2} \delta\left(\omega^{2}-M_{i}^{2}\right)+\frac{3}{8 \pi^{2}} \omega^{2} \theta\left(\omega^{2}-s_{0}\right)\left(a_{H}+b_{H} \frac{s_{0}^{2}}{\omega^{2}}\right) \sqrt{1-\frac{s_{0}^{2}}{\omega^{2}}}
$$

The mass parameters $M_{i}$ and wave function dependant decay constants $F_{i}$ for the $i^{t h}$ state of the charmonia are deduced from the medium dependant potential model adopted for the present study. The parameter, $s_{0}$ is chosen such that no resonance above it is possible. The calculated leading order coefficients $\left(a_{H}, b_{H}\right)$ are taken as $(-1,1),(1,0)$ and $(2,1)$ for scalar, pseudoscalar and vector channel respectively as given by [焑]. 

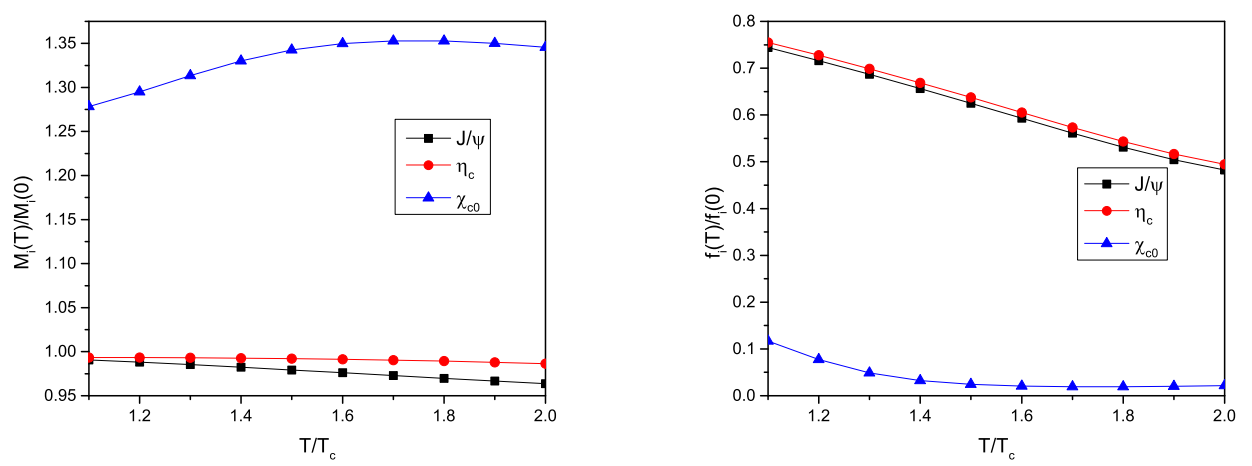

Figure 1: (a)Ratio of the mass and (b)Ratio of decay constant at given temperature to the zero temperature mass for different quarkonium states at different potential exponent $v$

To see the temperature effect on the spectral function and to compare with the lattice QCD results one usually computes the ratio of this correlators to the reconstructred one, $G(\tau, T) / G_{\text {recon }}(\tau, T)$, where $G_{\text {recon }}(\tau, T)$ is defined as,

$$
G_{\text {recon }}(\tau, T)=\int_{0}^{\infty} d \omega \sigma(\omega, T=0) K(\tau, \omega, T)
$$

Here, $G_{\text {recon }}(\tau, T)$ corresponds to the spectral function at the zero temperature. The temperature dependance in the $G_{\text {recon }}(\tau, T)$ comes only through the kernel $K(\tau, \omega, T)$.

\section{Extraction of spin average mass $\left(M_{i}\right)$ and decay constants $\left(F_{i}\right)$}

The spin average mass and decay constants appeared in the expression of the spectral function (Eqn. [2.3) are deduced using an appropriate model description of the hadronic state. Here, for the description of the quarkonia states, we consider temperature dependant screened potential of the form,

$$
V(r, T)=-\frac{\alpha}{r} e^{-\mu(T) r}+\frac{\sigma}{A \mu(T)}\left(1-e^{-A \mu(T) r^{v}}\right)
$$

Here, $\alpha$ and $\sigma$ are the coupling constant and string tension respectively. The exponent, $v$ represents the coulomb plus power law form of the potential at low temperatures. Different choices of the exponent, $v$ describes different strength of the interquark interaction. The screening mass parameter $\mu(T)$ is taken as, $\mu(T)=0.24+0.31\left(T / T_{c}-1\right) \mathrm{GeV}$ [四] with critical temperature $T_{c}=0.270$ $\mathrm{GeV}$. Here, we have chosen a parameter, $A\left(A=1 \mathrm{GeV}^{v-1}\right)$ for dimensional consideration. In the absence of the medium effect (at zero temperature) the potential in Eqn. 3.T reduces to the form $V(r)=-\alpha / r+\sigma r^{v}$. The parameters $\alpha, \sigma$ and the quark masses are fixed by fitting the zero temperature quarkonium spectrum as in [ [ $\left[\right.$, \&] and are given by $\alpha=0.471, \sigma=0.192 \mathrm{GeV}^{v+1}$ and $m_{c}=1.32 \mathrm{GeV}$. The spin average masses and the wave functions are obtained by solving the Schrödinger equation numerically using the screened potential of Eqn. B.D as quark-antiquark interaction potential for a chosen temperature. Computations are repeated for different choices of 

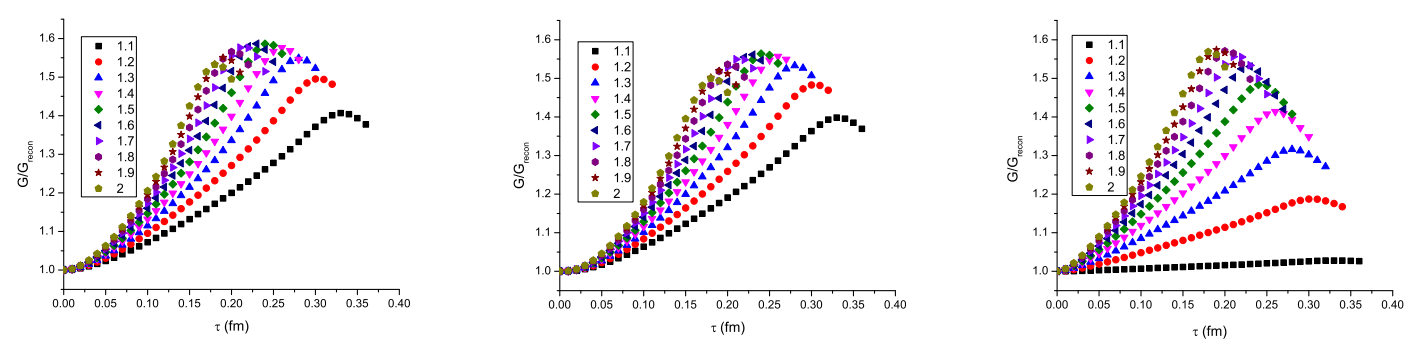

Figure 2: $G / G_{\text {recon }}$ for pseudoscalar charmonium states $(v=1.0)$ (a) $\eta_{c}$ state (b) $J / \psi$ state and (c) $\chi_{c 0}$ state

$T$.

Using the radial solution of the Schrödinger equation and by incorporating first order QCD correction to the Van Royen-Weiskopff formula, the decay constant is computed as [Q, प0],

$$
f_{P / V}^{2}(n S)=\frac{3\left|R_{n P / V}^{(\ell)}(0)\right|^{2}}{\pi M_{n P / V}}\left(1-\frac{\alpha_{s}}{\pi} \delta^{V, P}\right)
$$

Here $\delta^{V}=\frac{8}{3}[\mathbf{Q}, \mathbb{0}]$ and $\delta^{P}=2[9,[0, \square]$. To find the state dependant masses of charmonium states we follow the same procedure discussed in [[]]. The results are shown in fig. W in terms of quarkonia masses and its decay constants normalized to those at $T=0$ versus $T / T_{c}$.

Finally, the quarkonia correlators can be obtained by integrating Eqn.2.]. Our results for $G / G_{\text {recon }}$ are shown in Fig. $\square(\mathrm{a}-\mathrm{c})$ for pseudoscalar $\left(\eta_{c}\right)$, vector $(J / \psi)$ and scalar $\left(\chi_{c 0}\right)$ charmonium states respectively.

\section{Results and Discussion}

It is found from Fig. $\mathbb{W}$ that $M_{i}(T) / M_{i}(0)$ for i correspond to $J / \psi$ and $\eta_{c}$ decrease slowly with $T / T_{c}$. While $M_{i}(T) / M_{i}(0)$ for $\chi_{c 0}$ found to increase initially upto $T / T_{c}=1.7$ before it starts decreasing. But the decay constants $f_{i}(T) / f_{i}(0),\left(\mathrm{i}=\eta_{c}, J / \psi\right)$ found to decrease monotonically with increase in $T / T_{c}$, while $f_{i}(T) / f_{i}(0)$ for $\chi_{c 0}$ state is found to decrease upto $T / T_{c}=1.7$.

Our results of the charmonia correlators $G / G_{\text {recon }}$ for $\eta_{c}, J / \psi$ and $\chi_{c 0}$ states are shown in fig. 叉. It is very striking to see that $G / G_{\text {recon }}$ attains maximum at the same value of $\tau\left(\sim \tau_{\max }\right)$ for $\eta_{c}, J / \psi$ and $\chi_{c 0}$ states for a chosen $T / T_{c}$, and $\tau_{\max }$ found to decrease with increase of $T / T_{c}$.

The correlator $G / G_{\text {recon }}$ computed for $\eta_{c}$ (fig. $\square$ a) shows agreement with other potential model calculation [四]. Our results are in accordance with the QCD sum rule results [[13] at $T=1.1 T_{c}$, but differs from the lattice results [ㅁ] where $G / G_{\text {recon }}$ remains unity upto $\tau \approx 0.05 \mathrm{fm}$ and it systematically decreases as $\tau$ increases.

In the case of $G / G_{\text {recon }}$ for vector charmonium state $(J / \psi)$, present result is in agreement with the lattice results [ए2] and also with the QCD sum rules [[13] for smaller value of $\tau$. Though the present $G / G_{\text {recon }}$ in the case of $\eta_{c}$ increases from 1.0, the results based on fine isotropic lattice study shows decreasing effects from $T=T_{c}$ for all temepratures $T>T_{c}$. Our results of $G / G_{\text {recon }}$ for scalar

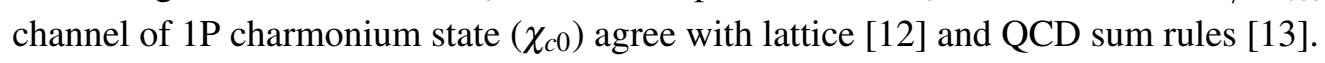


The $\tau_{\max }$ representing the temperature for the maximum correlation of the quarkonia states found to be exponentially decreasing with the temperature in the case of charmonium states. The values of $G / G_{\text {recon }}$ below 1 means the survival probability of the state is weaker and leads to dissociation of the quarkonium state. Considering the value of $\tau$ for which $G / G_{\text {recon }} \leq 1$ and associating a temperature, $T_{d}$ related to $\tau_{d}$ as $\tau_{d}=1 / T_{d}$ we obtain the dissociation temperature of $\eta_{c}, J / \psi$ and $\chi_{c 0}$ states as $\sim 1.2 T_{c}$.

\section{References}

[1] Agnes Mocsy and Peter Petreczky, Phys. Rev. D 73074007 (2006).

[2] Gert Aarts et al., Phys. Rev. D 76094513 (2007).

[3] R. Rappa, D. Blaschke and P. Crochet, Prog. Part. and Nucl. Phys. 65209 (2010).

[4] Agnes Mocsy and Peter Petrezsky, J Phys. G 32 S515 (2006).

[5] E V Shuryak, Rev. Mod. Phys. 651 (1993).

[6] F. Karsch, M.G. Mustafac and M.H. Thoma, Phys. Lett. B 497249 (2001).

[7] S Jacobs, M G Olsson and C I Suchyta, Phys. Rev. D 333338 (1986); 343536 (E) (1986).

[8] Arpit Parmar, Bhavin Patel and P C Vinodkumar, Nucl. Phys. A 848299 (2010).

[9] Berezhnoy A V, Kiselev V V and Likhoded A K, Z. Physik A336, 89 (1996).

[10] Braaten E and Fleming S, Phys. Rev. D52, 181 (1995).

[11] S S Gerstein et al., arXiv:hep-ph/9803433v1.

[12] H.-T. Ding et al., Phys. Rev. D 86014509 (2012).

[13] Kenji Morita and Su Houng Lee, Phys. Rev. D 82054008 (2010). 